\title{
Performance Evaluation of LTE Scheduling Techniques for Heterogeneous Traffic and Different Mobility Scenarios
}

\author{
Lukmanhakim Sukeran , Mohamed Hadi Habaebi ${ }^{1}$, Al-Hareth Zyoud, Musse \\ Mohamud Ahmad, Shihab Hameed, Amelia Wong, MD. Rafiqul Islam \\ Electrical and Computer Engineering Department, Faculty of Engineering, \\ International Islamic University Malaysia (IIUM), \\ 53100 Gombak, Kuala Lumpur, Malaysia \\ Email: ${ }^{1}$ habaebi@iium.edu.my
}

\begin{abstract}
In this paper five scheduling algorithms were investigated and their performance was evaluated in terms of Fairness Index, Peak Throughput, Average Throughput and Edge Cell User Throughput. A system level MATLAB simulator was used. The simulation takes into account different types of traffic for several mobility scenarios and propagation channel models. Results indicate that the scheduling algorithms showed some quality in certain parameter of evaluation but lack in other terms. While some scheduling algorithm take the moderate path but still be lacking especially in Edge Cell User Throughput necessitating the use of Relays or femtocells.
\end{abstract}

\section{Introduction}

Evolution of Universal Mobile Telecommunications System (UMTS) has not reached its end even though with the existence of High Speed Packet Access (HSPA). UMTS Long Term Evolution (LTE) has been introduced in 3rd Generation Partnership Project (3GPP) Release 8 to guarantee the competitiveness of UMTS for the next coming years. The rapid grow of mobile data usage in the recent years such as gaming, mobile channel TV, and other streaming content have concerned in the (3GPP) leading to motivation on LTE. Therefore the work towards 3rd Generation Partnership Project (3GPP) Long Term Evaluation started in 2004 and the targets of LTE standard were set [1].

Orthogonal Frequency Division Multiplexing (OFDM) has been adopted as the downlink transmission scheme for the 3GPP LTE. OFDM is a multicarrier transmission scheme since it splits up the transmitted high bitstream signal into different sub-streams and sends these over many different subchannels [2]. OFDM simply divides the available bandwidth into multiple narrower sub-carries and transmits the data on these carries in parallel streams. Each subcarrier is modulated using different modulation scheme, e.g. Quadrature Phase Shift Keying (QPSK), Quadrature Amplitude Modulation (QAM), 64QAM and an OFDM symbol is obtained by adding the modulated subcarrier signals [3]. 
The scheduling algorithm is the radio resource management technique that is used by the base station to manage and control the available radio resources and assign them efficiently to the available users to meet their service requirement. The minimum resources that could be assigned for a user are called Resource Block (RB). RB includes 12 adjacent OFDM subcarriers. The scheduler task is to assign these RBs to the users in the network. Many scheduling algorithms have been proposed in the literature. So far, Different studies have been conducted to investigate the performance of the proposed algorithms for different scenarios using several simulation platforms.

The work in [4] investigated the performance of five scheduling algorithms for video traffic using 3GPP LTE simulator. The results showed that Maximum-Largest Weighted Delay First (M-LWDF) algorithm performs better than other algorithms like Round Robin (RR), Exponential/Proportional Fair (EXP/PF), Maximum Rate (Max-Rate), and Proportional Fair (PF) in terms of throughput, number of users supported and fairness. In [5], a comparison of different scheduling algorithms for downlink channel was performed using NS-3 simulator. Similarly, NS-3 was used in [6] to evaluate the performance of scheduling algorithms for uplink scenarios. Moreover, LTE-Sim [7] was used in [8] to compare the performance of three different scheduling algorithms in video traffic scenarios. Habaebi et al [9] evaluated three of the most known scheduling algorithms namely, RR, PF and Best Channel Quality Indicator (BCQI) using LTE system level simulator [10]. They found that the BCQI outperforms RR and PF in terms of throughput and Block Error Rate (BLER).

In this paper five types of scheduling are considered which are RR, Proportional Fair Sun (PFS), BCQI, Resource Fair Maximum Throughput (RF) and Max-Min Fairness (MaxMin). System level simulations were carried out to compare and evaluate the previous algorithms. The performance was evaluated in terms of fairness index, peak throughput, average throughput and cell edge throughput.

\section{Scheduling Algorithms}

The scheduling algorithms that are investigated in this paper are highlighted in the sub sections below:

\subsection{Resource Fair Maximum Throughput (RF)}

Resource Fair Maximum Throughput algorithm integrates the Max-rate and Proportional Fair scheduling. This algorithm efficiently employs available radio resource as user's packets are transmitted on a radio resource with a good channel condition. Users are treated according to the rank and the schedulers are either Maxrate of Proportional Fair [11]. 


\subsection{Proportional Fair Sun (PFS)}

This scheduling algorithm is basically the improvement and less complexity form of the PF scheduling algorithm. Karush-Kuhn-Tucker (KKT) condition was used to reduce the complexity of the PF [12].

\subsection{Max-Min Fairness (MaxMin)}

The algorithm key parameter is fairness. It distributes the resource block to achieve optimal fairness. It aims to provide the maximum resource to the minimum date rate for the receiver so that the data rate distribution is fair [11].

\subsection{Best Channel Quality Indicator (BCQI)}

BCQI scheduling policy is to allocate resource blocks to the user with the best channel condition. In order to perform scheduling, terminals send Channel Quality Indicator (CQI) to the base station [13].

\subsection{Round Robin (RR)}

$\mathrm{RR}$ is proposed to solve the problem of fairness that appears in the BCQI and RF algorithms. It is allocate equal time for each user without priority option. Therfore, the channel condition has no impact on the user chance. Basically this algorithm rate the user in term of first come first serve basis [13]. The fairness is improved, however the throughput is degraded signifantly.

\section{Simulation Environment}

In this paper, 7 hexagonal base stations (eNodeBs) with various number of user equipments (UEs) are used. The users are randomly located in the eNodeB region of interest. The LTE system level simulator parameters are given in Table I. The mobility is considered in all scenarios (pedestrian and vehicular). Five different traffic types have been considered: VoIP, Video, FTP, HTP, and Gaming.

\section{$4 \quad$ Simulation Results and Discussions}

Using the parameters presented in Section 3, the results were generated for different traffic types, for each mobility scenario and for various numbers of users. The figures in this section show the generated result for VoIP service only since there is no enough space to show all the generated results for all traffic types. However, all results were discussed at the end of the section. The results for VoIP traffic for pedestrian UE with speed 3 km/h are presented in Fig. 1- Fig. 4. 
Table 1. Simulation parameters for LTE system level simulator.

\begin{tabular}{ll}
\hline Parameter & Value \\
\hline Frequency & $2.14 \mathrm{GHz}$ \\
Bandwidth & $20 \mathrm{MHz}$ \\
Simulation length & $100 \mathrm{TTI}$ \\
Inter eNodeB distance & $500 \mathrm{~m}$ \\
eNodeB TX power & $20 \mathrm{dBW}$ \\
Number of UEs & $1,2,5,10,20,40$ users \\
Antenna pattern & Omni-directional \\
eNodeB antenna gain & $15 \mathrm{dBi}$ \\
Uplink delay & $3 \mathrm{TTIs}$ \\
UEs Speed & $3 \mathrm{Km} / \mathrm{h}$ and $120 \mathrm{Km} / \mathrm{h}$ \\
\hline
\end{tabular}

In terms of fairness index as in Fig. 1, BCQI shows declination rapidly compares to other scheduling algorithms as the number of users increase. Other scheduling algorithms have uniform distribution of fairness index ranging in between the values of 0.64 and 0.87 . However, In terms of peak throughput as in Fig. 2, BCQI scores the highest value of peak throughput $160 \mathrm{Mbps}$ and this value is maintain up to 2 users before it dropped. Other scheduling algorithms showed decreasing trend as the number of user increase and only has the highest Peak Throughput at the smallest number of user which is 1 .

For average throughput as in Fig. 3, all the scheduler showed a somewhat same behavior. Average throughput decreases as the number of user increases. BCQI comes to have the highest value in throughout, while other scheduling algorithms are having about the similar values. For the edge users as in Fig. 4, PFS comes out as the best for 1 to 2 users before the MaxMin takes the lead for 5 and above number of users. On the other hand, BCQI only treats the edge user when there is only 1 user while as the number of user increases to 2 , the edge users are no longer experiencing throughput.

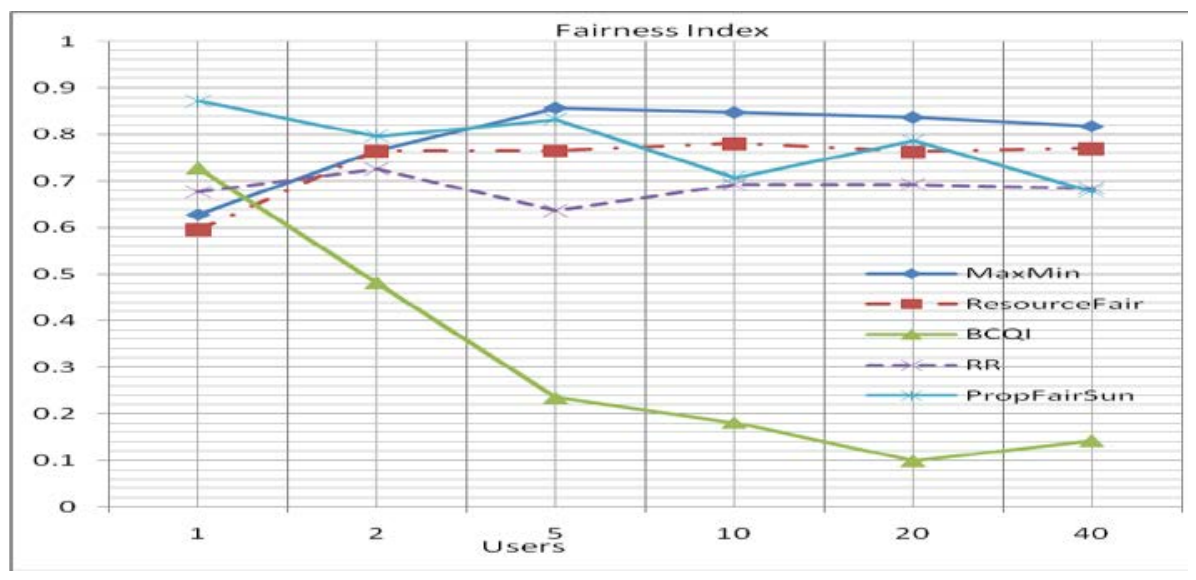

Fig. 1. Fairness index against number of users. 


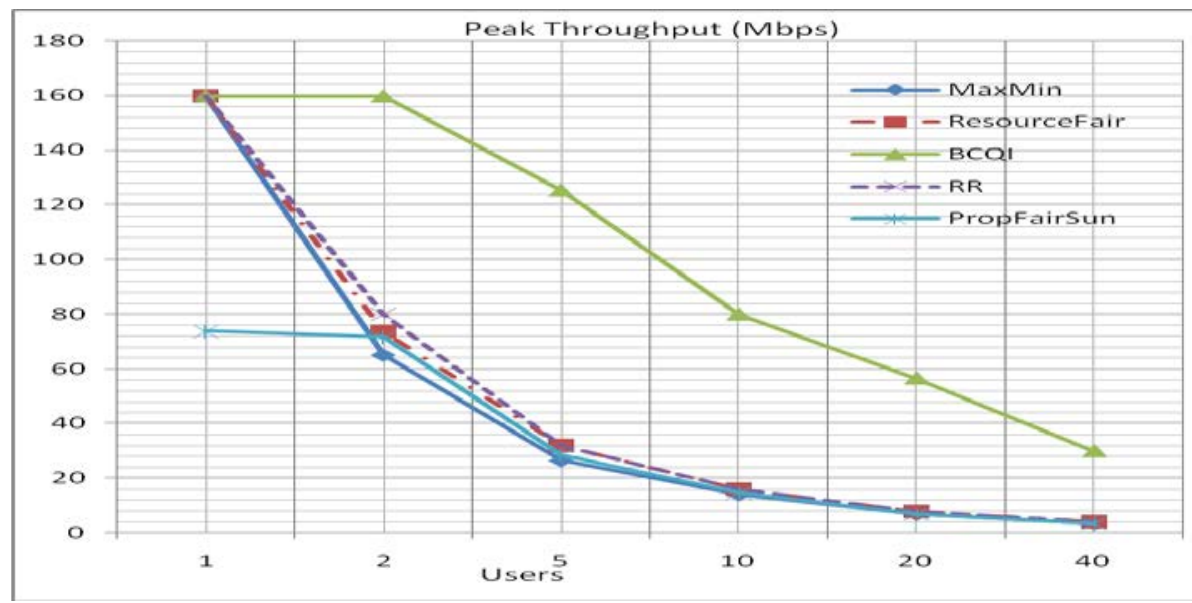

Fig. 2. Peak throughput against number of users.

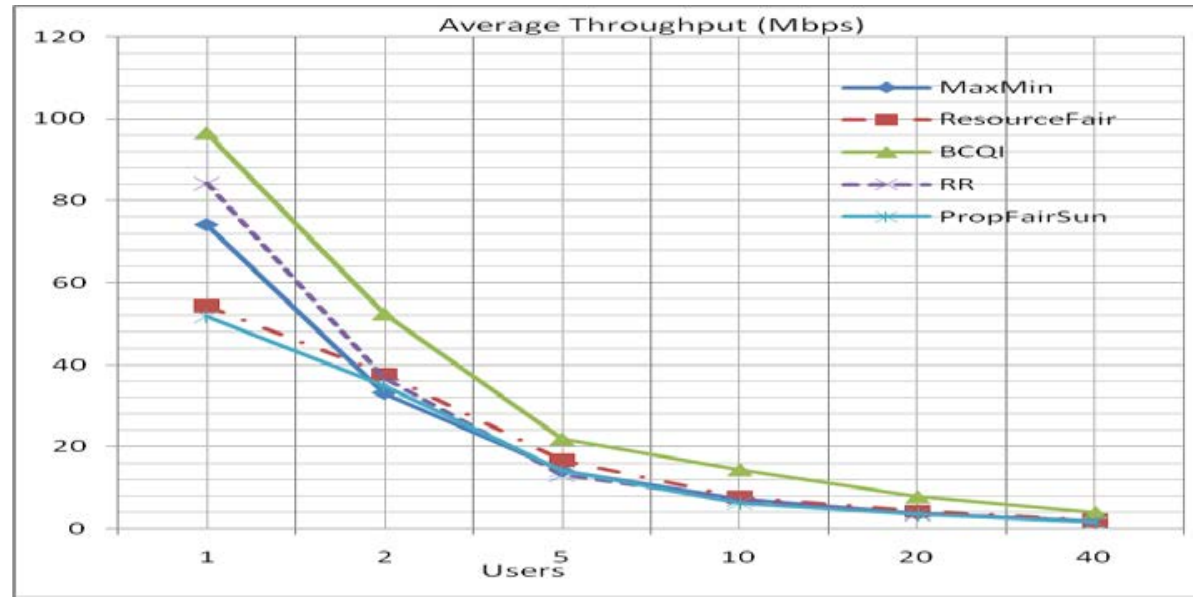

Fig. 3. Average throughput against number of users. 


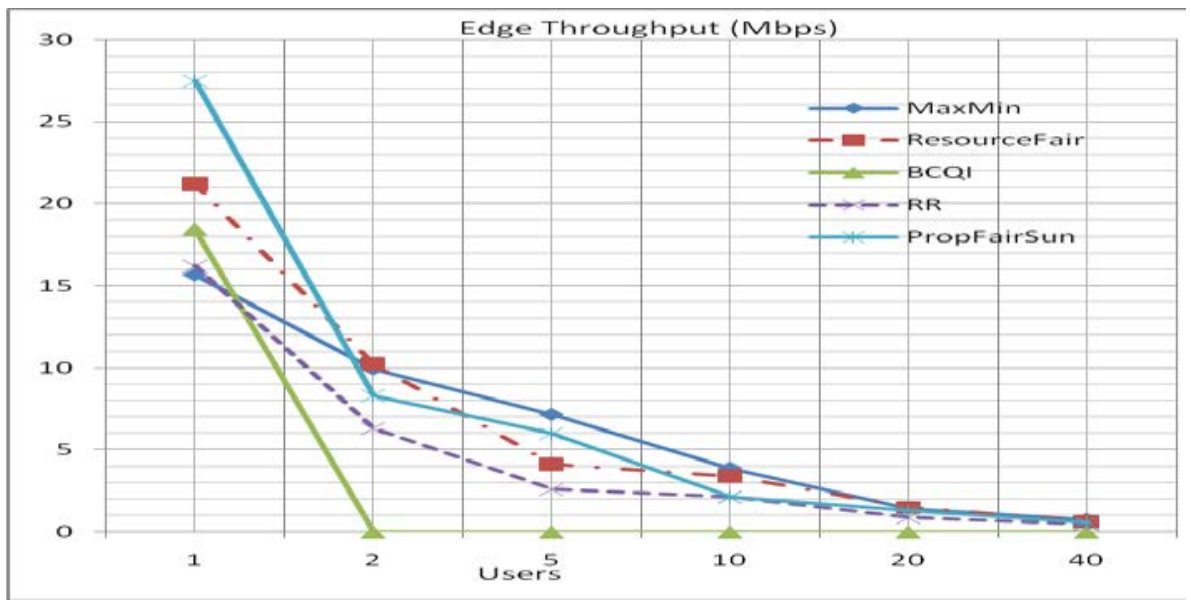

Fig. 4. Edge user throughput against number of users.

In the second scenario, the speed of the UE was changed to be $120 \mathrm{Km} / \mathrm{h}$ for VoIP service. Fig. 5 shows the fairness as a function of number of users. MaxMin has the highest value among other schedulers in case of 1and 2 UEs. Then Round Robin took the place as the number of users increase from 3 to 40. Round Robin has the smallest decrement of fairness index compare to other schedulers. Same as the first scenario, BCQI has the lowest value among the other schedulers. However, in terms of peak throughput as in Fig. 6, BCQI able to achieve the highest throughput value at $160 \mathrm{Mbps}$ and maintain it until 2 users. PFS also able to achieve the value of 160Mbps but dropped sharply as the number of user increase from 1 to 5 users and above. Other schedulers show almost the same behavior.

In addition, BCQI shows the best performance in terms of average throughput as in Fig. 7. The other schedulers have about the similar value to each other. All the schedulers showed the same trend as the value of Average Throughput decrease exponentially as the number of user increases from 1 to 40 in the cell.

Finally, for the edge users as in Fig. 8, RR and RF are able to support more than 5 users at the cell edge. BCQI shows the worst case where only can support 1 user. 


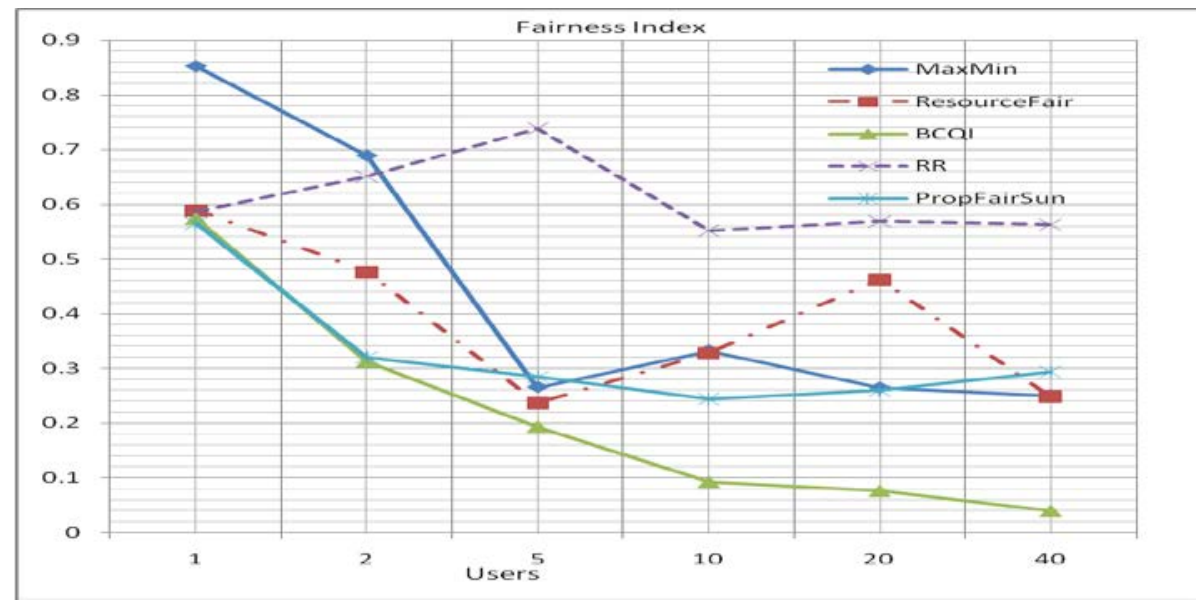

Fig. 5. Fairness index against number of users.

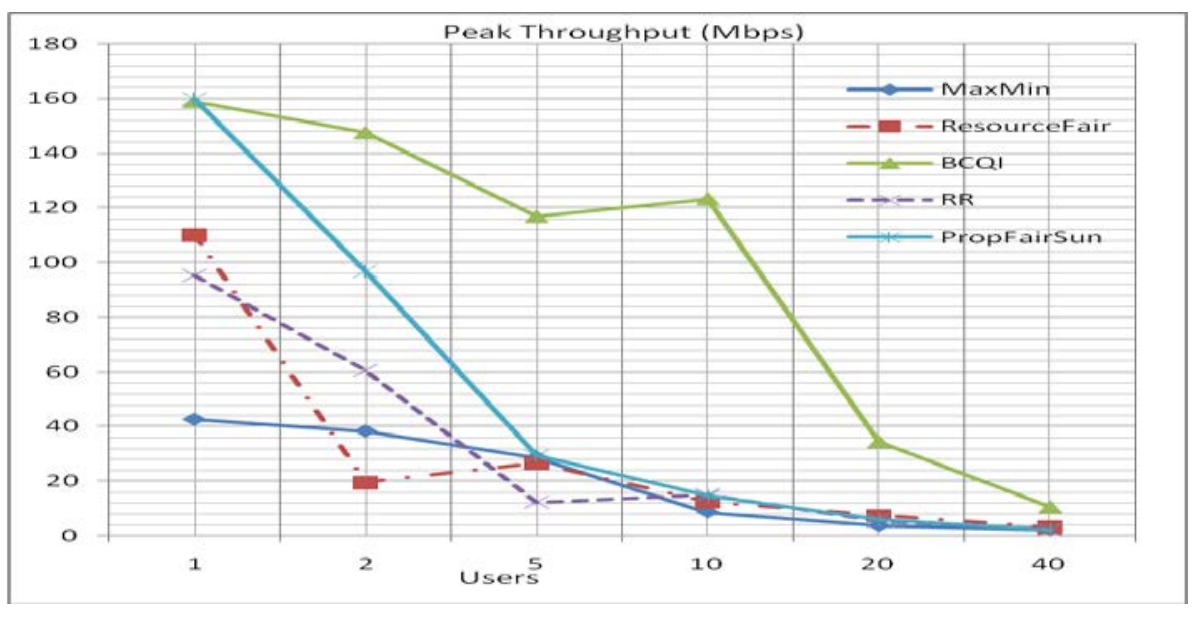

Fig. 6. Peak throughput against number of users. 


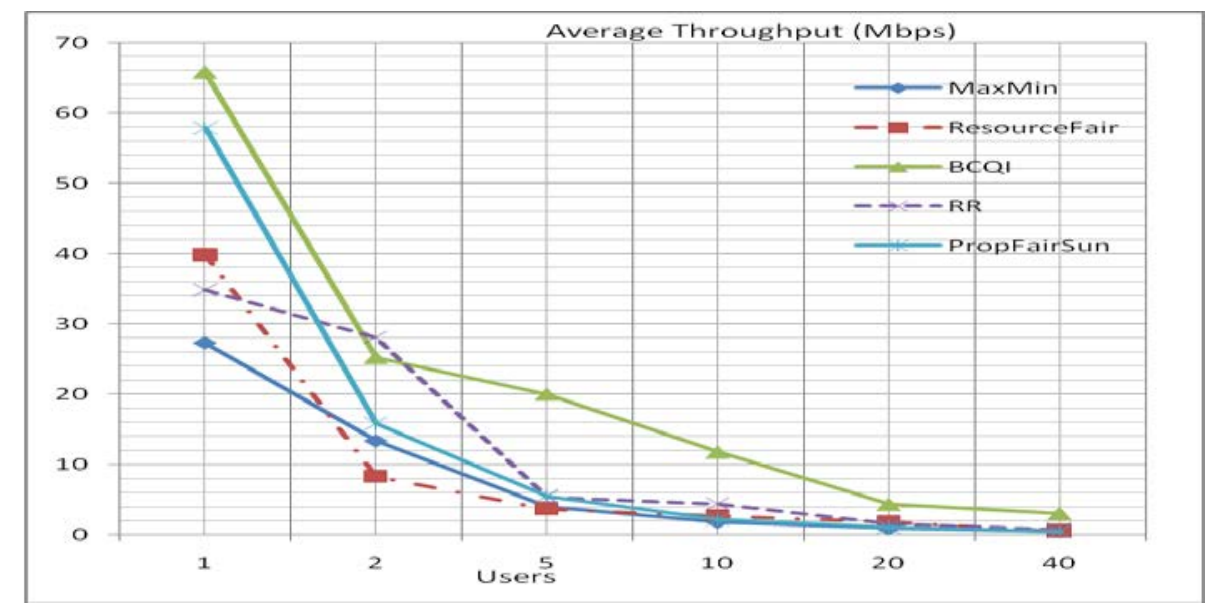

Fig. 7. Average throughput against number of users.

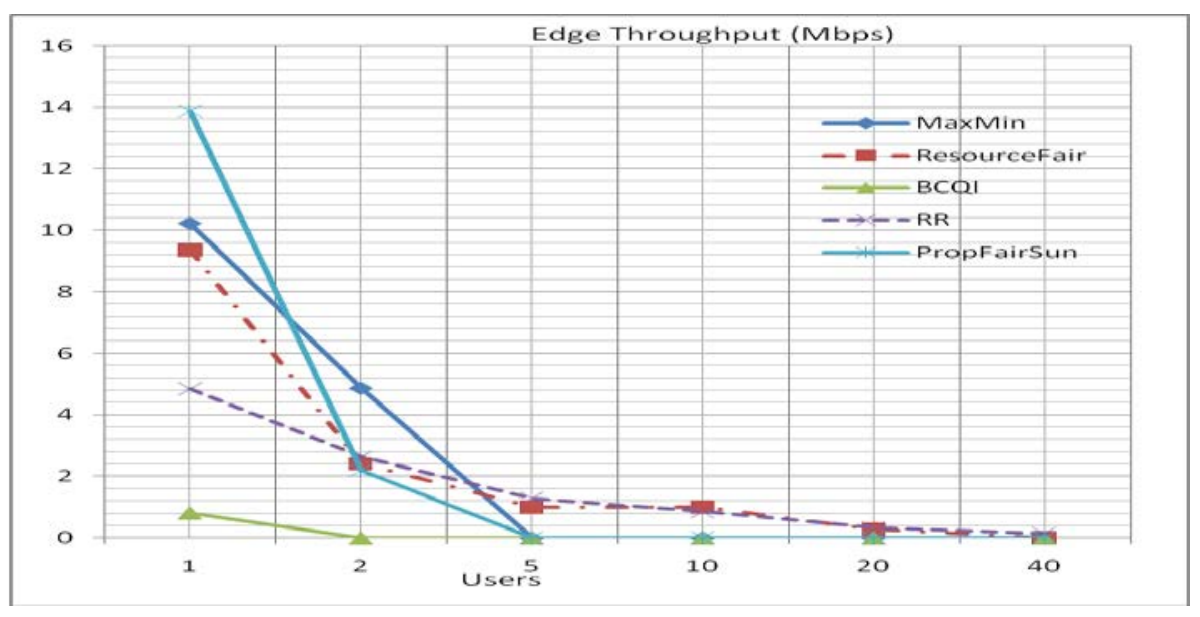

Fig. 8. Edge user throughput against number of users.

In all the traffics types' scenarios, generally, all the results show that the throughput decreasing as the number of users increasing, same goes for the edge cell UE. Both of these parameters have inversely proportional relationship to each other. However in terms of the fairness index, RR algorithm able to maintain the fairness index value as the number of users increase in several scenarios. In pedestrian and vehicular scenarios, values of fairness index can be considered as stagnant and having 
fixed range except for BCQI algorithm. However, BCQI performs very well in terms of peak and average throughput.

To sum, BCQI is not suitable for real-time transmission since it seems to cannot provide the QoS for the VoIP when incomes to cell edge users compare to the other scheduler especially Round Robin, the reason behind that is the channel quality for the edge user is worse than the cell center users and using BCQI scheduler the edge user will not be able to get a good service.

In terms of efficiency, the BCQI can be seen as the most efficient scheduler compare to the other scheduler due to the peak throughput achieved has the highest value show that the spectral efficiency is the highest while, in term of effectiveness, Round Robin is the most effective scheduler since it still provide throughput to the edge users and for high number of users, since RR allocates equal time and data rate for each user in the network. The other scheduler performed moderately.

\section{Conclusion}

All the proposed scheduling algorithms; RR, PFS, RF, MaxMin, and BCQI has been investigated. Fairness index, peak throughput, average throughput, and edge users throughput also has been achieved and the entire scheduling algorithm has been tested for several traffic types. Each one of the scheduling algorithm has shown performance merit in certain criteria of evaluation. For instance, RR has shown that it is good for vehicular channel model, while BCQI has shown the best in achieving peak throughput. However, the best suited scheduling algorithm is still remains argumentative and in need for extensive and more comprehensive improvement not just the scheduling algorithm but the system infrastructure as a whole and also the simulation platform. However, the use of cell edge Relays and Femtocells is necessary.

Acknowledgments. This work was funded by E-Science grant 01-01-08-SF0194 from Malaysian ministry of Science, Technology and Innovation (MOSTI).

\section{References}

1. 3GPP,: Evolved Universal Terrestrial Radio Access (E-UTRA) and Evolved Universal Terrestrial Radio Access Network (E-UTRAN); Overall description; Stage 2. TS 36.300, 3rd Generation Partnership Project (3GPP), (2010).

2. Myung, H. G., Goodman, D.,: Single Carrier FDMA, New Air Interface for LTE. Wiley, (2008).

3. Goldsmith, A.,: Wireless Communications. Cambridge University Press (2005).

4. Ramli, H., Basukala, R., Sandrasegaran, K., Patachaianan, R.,: Performance of Well Known Packet Scheduling. in Proceedings of the IEEE 9th Malaysia International Conference on Communications , Kuala Lumpur, (2009), pp. 815-820.

5. Zhou, D., Baldo, N., Miozzo, M.,: Implementation and validation of LTE downlink schedulers for ns-3. In Proceedings of the 6th International ICST Conference on Simulation Tools and Techniques (SimuTools '13). ICST (Institute for Computer Sciences, Social- 
Informatics and Telecommunications Engineering), ICST, Brussels, Belgium, Belgium, (2013) pp. 211-218.

6. Safa H., Tohme, K.,: LTE Uplink Scheduling Algorithms: Performance and Challenges. 19th International Conference on Telecommunications (ICT), (2012) pp. 1-6.

7. Piro, G., Grieco, L.A., Boggia, G., Capozzi, F., Camarda, P.,: Simulating LTE Cellular Systems: An Open-Source Framework. IEEE Transaction on Vehicular Technology, Vol. 60, No. 2. (2011).

8. Sahoo, B.,: Performance Comparison of Packet Scheduling Algorithms for Video Traffic in LTE Cellular Network. International Journal of Mobile Network Communications \& Telematics ( IJMNCT) Vol. 3, No.3, June (2013).

9. Habaebi M. H., Chebil J., Al-Sakkaf A.G., and Dahawi T. H.,: Comparison between Scheduling techniques in long term evolution. IIUM Engineering Journal Vol. 14, No. 1, (2013) pp 66-75.

10. Ikuno, J.C., Wrulich, M., Rupp, M.,: System level simulation of LTE networks. in Proc. 2010 IEEE 71st Vehicular Technology Conference, Taipei, Taiwan, May (2010). [Online] Available: http://publik.tuwien.ac.at/files/PubDat_184908.pdf

11. Manor R., Romsey,: LTE MAC Scheduler \& Radio Bearer QoS. (2011).

12. Sun, Z., Yin, C., Yue, G.,: Reduced-Complexity Proportional Fair Scheduling for OFDMA Systems. in Proc. IEEE International Conference on Communications, Circuits and Systems, vol. 2. (2006).

13. Dahlman, E., Parkvall, S., Skold, J., Beming, P.,: 3G Evolution HSPA and LTE for Mobile Broadband. 2nd edn, Elsevier, (2008). 